\title{
The Cultural Evolution of Adaptive-Trait Diversity when Resources are Uncertain and Finite
}

\author{
M.W. Lake \\ E.R. Crema \\ UCL Institute of Archaeology \\ mark.lake@ucl.ac.uk
UCL Institute of Archaeology
e.crema@ucl.ac.uk \\ Authors' postprint of an article published in Advances in Complex \\ Systems 15: 1150013-1-1150013-19; DOI: \\ 10.1142/S0219525911003323; (C)World Scientific Publishing \\ Company; http://www . worldscientific.com/worldscinet/acs
}

\begin{abstract}
In this paper we seek to build on existing mathematical studies of cultural change by exploring how the diversity of adaptive cultural traits evolves by innovation and cultural transmission when the payoff from adopting traits is both uncertain and frequency dependent. The model is particularly aimed at understanding the evolution of subsistence trait diversity, since the payoff from exploiting particular resources is often variable and subject to diminishing returns as a result of overexploitation. We find that traits that exploit the same shared resource evolve most quickly when intermediate rates of cultural transmission promote fluctuation in trait diversity. Higher rates of cultural transmission, which promote predominantly low diversity, and lower rates, which promote predominantly high diversity, both retard the adoption of traits offering higher payoff. We also find that the distribution of traits that exploit independent resources can evolve towards the theoretical Ideal Free Distribution so long as the rate of cultural transmission is low. Increasing the rate of cultural transmission reduces trait diversity, so that a more limited number of 'niches' are occupied at any given time.

KEYWORDS: Cultural Transmission; Innovation; Cultural Diversity; Subsistence Strategies; Agent Based Modelling.
\end{abstract}

\section{Motivation}

The study of cultural change using concepts and mathematical tools borrowed from the study of biological evolution is now well established [24], but as Kandler and Laland [12] have recently observed, the relationship between innovation and cultural diversity has received less attention, even though it is central to 
the history of anthropological thinking about how and why human behaviour changes. In this paper we explore how innovation and cultural transmission influence cultural diversity in adaptive traits when the payoff obtained from adopting those traits is both uncertain and regulated by population dynamics.

Our interest in this problem stems from the fact that a central theme in the archaeological investigation of human history is the evolution of subsistence strategies, which can be modelled in terms of changing adaptive trait diversity in at least two senses. One of these is nicely encapsulated in Layton et als' [13] argument that hunting and gathering, herding, and cultivation should be treated as different adaptive strategies rather than as an "evolutionary progression from one distinct type of society to another". The difficulty of maintaining the earlier social-evolutionist position is easily demonstrated by pointing to a number of cases in which it appears that groups have switched strategies at least once and in some cases repeatedly. For example, Schrire [23] has argued that some San communities have oscillated between foraging and cattle herding over several centuries or even millennia, while Mace [16] lists a number of instances of groups switching back and forth between pastoralism and farming in the Sudano-Sahelian belt. More recently, Oota et al [20] have presented genetic, linguistic, and cultural evidence that the Thai Mlabri adopted hunting and gathering despite having originated from an agricultural group, while in east Africa the Dorobo, Masaai and Kikuyu are known to revert to hunting and gathering after crop failure [11]. Layton et al [13] argued that switching between different subsistence strategies could usefully be conceptualised in terms of the optimal diet-breadth model $[15,22]$ because it explains when people would be expected to switch from resources offering a high net energetic return after search and handling costs (typically animal foods) to those offering a lower return (typically plant resources).

The second sense in which evolution of subsistence systems can be modelled in terms of changing adaptive trait diversity arises from the invocation of the optimal diet-breadth model to explain the range of prey species exploited by hunter-gatherers. Three basic predictions [22] of the diet-breadth model are that: i) the decision whether to incorporate a food item into the diet is determined by the abundance of higher-ranking food types rather than its own encounter rate; ii) an increase in search efficiency will result in a reduction of diet breadth; iii) an increase in pursuit efficiency will result in an expansion of diet breadth. Behaviour consistent with all three predictions has been observed in empirical studies. For example, Hames and Vickers [10] found that the Amazonian Siona-Secoya, Ye'kwana, and Yanomamb incorporated food types in their diet in rank order of net energetic return, but that the range of foods exploited increased after their acquisition of guns reduced pursuit time. In contrast, Winterhalder [30] observed that the acquisition of snowmobiles by the North American Cree greatly increased their search efficiency thereby allowing them to concentrate more narrowly on higher ranked larger game offering a higher rate of energetic return.

It is clear that the optimal diet-breadth model can explain at least some specific examples of the decision to hunt new types of prey and even the switching 
back-and-forth of modes of subsistence. More generally, however, its application to human societies has lead to a better understanding of the complex relationship between resource scarcity, technological innovation, diet breadth and population size. The first lesson is that even purely selfish decision-making can prevent the extinction of preferred resources due to overexploitation provided that the declining return from those resources (due to increased search time) is eclipsed by the return from lesser ranked resources before extinction of the former [26]. The second lesson is that because technological innovation directly influences search and/or handling costs it is often critical in determining which or two or more resources offers the greatest net energetic return [10].

Despite its demonstrable utility, however, it is notable that applications of the diet-breadth model typically relate to subsistence changes occurring over what one might call an ethnographic time-scale (but see [32]). Specifically, applications of the diet-breadth model essentially assume that the people already know the net-energetic return from all the resources available to them, in other words, there is no need for technological innovation or it just 'happens'. Given our archaeological interest in explaining change over the long-term we are acutely aware that subsistence trait diversity at any given time must be a function of both the type of rational decision-making captured by the dietbreadth model and the preceding history of subsistence technology. Moreover, we suspect that the relationship between these forces is complicated by at least two factors: i) that reliable assessment of a new technology is likely to be very dependent upon the predictability of the resource that it is designed to exploit; and ii) that, as Shennan [25] has demonstrated, new technologies are more likely to be lost in smaller populations. The model we present in this paper is intended to help us explore how these factors influence change in adaptive trait diversity in evolutionary time, that is to say, over timescales where the adoption or loss of innovations in subsistence strategy is itself part of the problem.

Our model of the evolution of adaptive trait diversity builds on the work of Kandler and Laland [12] and Shennan [25]. The well-known explosion of cultural diversity at the so-called Middle-Upper Palaeolithic transition in Europe[17] motivated Shennan [25] to develop a mathematical model of the innovation and spread of fitness enhancing craft skills. He modelled the fitness of an individual as the multiplicative sum of fitnesses of each of a fixed number of craft attributes and then allowed innovation to alter the fitness of each attribute. Cultural transmission resulted in an individual copying the trait from the model with highest fitness for that trait among an 'effective' population. A series of simulation experiments revealed that fitness enhancing innovation is restricted by deleterious sampling effects in small populations, but significantly enhanced by cultural transmission in larger populations. As a result Shennan (p.13) noted that "advantageous cultural change is at least as much a consequence as a cause of increased population", but because his model parameterises population size he was not able to more formally explore the feedback between innovation and population growth. In this paper we extend Shennan's analysis in that direction by allowing traits to determine the population growth rate. A further interesting question raised by Shennan's work stems from his observation that "it is 
highly unlikely that an individual would always be able to pick out the most beneficial older individual to copy". We incorporate this effect in our model by introducing payoff variance.

Kandler and Laland [12] have recently presented a reaction-diffusion model which describes the spread and changing diversity of competing cultural traits within a growing population. They model the evolution of " $n$ competing (mutually exclusive) variants of a specific cultural trait within a population" (p.60) where different variants confer different benefits on those who adopt them. New variants arise by improvement of an existing variant or by independent invention, while the adoption of existing variants results from unbiased social learning or various kinds of cultural transmission bias, including conformist bias. Kandler and Laland's primary interest is in the interplay between innovation and cultural transmission and they report several interesting results which include oscillating trends in diversity when innovation occurs by improvement as well as the negative impact of conformity on diversity. While our interest overlaps (indeed, we replicate some of their findings, as noted later) our emphasis is more on issues that arise directly out of our explicit concern with diversity in subsistence strategies. For that reason we follow Shennan [25] in modelling just one type of cultural transmission (payoff-biassed) and then focus our effort on the effects of payoff variance and alternative models of competition between strategies.

The problem of estimating the true value of a resource is well known in the optimal foraging literature [28] and has even been addressed in computer simulation experiments stimulated by archaeological studies of subsistence behaviour $[18,5]$. When chance events cause the actual payoff from exploiting a given resource to fluctuate around the mean payoff individual organisms may struggle to identify the true value of that resource. Moreover, when organisms copy subsistence strategies from one another there is the possibility that they may erroneously adopt a bad strategy or reject a good one, which may in turn have unexpected consequences if the payoff from that strategy is frequency dependent. For these reasons we take a different approach from Kandler and Laland, who assume a fixed payoff; instead we model payoff as a stochastic variable such that the payoff obtained by adopting a particular subsistence strategy is drawn from a probability distribution associated with that strategy.

In Kandler and Laland's model individuals with different traits compete for a share of a single set of resources. This is of interest to us since it models the situation where, for example, an individual innovates a new technology for exploiting a resource already used by the group (such as the case of the snowmobile cited above). However, there is another scenario which is also relevant in the context of subsistence: that where an individual innovates a new technology which enables the exploitation of a new resource, either because it makes it physically possible, or because it changes the cost/benefit calculation of the value of that resource. In the first case there is direct competition between the old and new subsistence strategies, but in the latter that is not the case. We model both scenarios since they occur in applications of the diet-breadth model to real-world ethnographic case studies. 


\section{The model}

Our model is designed to explore how the balance of innovation and payoffbiassed transmission influences cultural trait diversity when the payoff from the adoption of a specific trait has negative frequency dependence and is affected by some degree of stochasticity. The cultural traits are 'adaptive' in the sense that we envisage them being tools, technologies or strategies for exploiting subsistence resources. We implement two variants of the model. In the $S$-mode model agents with different traits compete for a share of a single resource pool, which captures the real-world situation where, for example, an individual innovates a new - possibly more efficient - technology for exploiting a resource already used by the group. In the I-mode model agents with different traits exploit separate resource pools, in this case capturing the real-world situation where an individual innovates a new technology which enables the exploitation of a previously untapped resource.

We implement our model as a discrete agent-based simulation (ABM). The strengths and weaknesses of ABM versus continuous mathematical models have been rehearsed elsewhere [7, 27, 14]; for our purposes the main benefit of using an ABM is greater sensitivity to the risk of trait loss in small populations. Our model implements a population of agents characterised by the following four state variables: age $(a)$, most recent payoff $(\phi)$, stored energy $(E)$ and adopted trait $(j)$. Each run of the simulation is initialised by the creation of $N$ agents possessing identical initial state variables (apart from $a$ ). The state variables are updated through a scheduled sequence of processes iterated through $T$ timesteps. At each time-step the payoff $\phi$ of each agent is evaluated. This modifies the amount of stored energy $E$ which in turn determines the probability of the agent's reproduction and survival. Each agent has the opportunity to modify its trait $j$ via innovation (with frequency $\mu$ ), or cultural transmission (with frequency $z$ ). Full details of the model can be found in the online supplementary material; here we describe the main processes which drive the dynamics.

The core process of the model is the computation of the payoff $\left(\phi_{i}\right)$ received by each agent $i$. This involves two stages. First, for each agent, a random

number $\omega_{i}$ is drawn from a normal distribution with mean $f_{j}$ and standard deviation $v_{j}$, where $j$ is an index value pointing at one of the $\theta$ possible traits that can be adopted. Each trait has a different mean, with a higher index value determining a higher expected payoff $f$, so that if $x>y$, then $f_{x}$ will be always greater than $f_{y}$. Variance is identical for all traits and is determined by the global parameter $v$. The subsequent step is different for the $S$-mode model and the I-mode model. In the former case the sum of all $\omega(\Omega)$ is computed and compared to $K$, the size of the shared resource pool. When $\Omega \leq K, \phi_{i}=\omega_{i}$, while when $\Omega>K$, the agents are overexploiting the resource and the payoff of each individual will be evaluated as follows:

$$
\phi_{i}=K \frac{\omega_{i}}{\Omega}
$$

In the I-mode model the same equation is applied independently for each 
trait. Thus $\Omega$ will be $\Omega_{j}$ which is the sum of $\omega$ of all agents having the trait $j$, while the resource pool is also trait specific ( $K_{j}$ instead of $K$ ).

For both modes, after $\phi$ is computed, the stored energy of each agent $\left(E_{i}\right)$ is updated by adding $\phi_{i}$ and then subtracting $c$, the latter representing the resource consumption rate. Thus, when $\phi_{i}-c>0, E_{i}$ will increase, while when $\phi_{i}-c<0, E_{i}$ will decrease. The stored energy plays a central role in determining the reproductive fitness of each agent. When $E_{i} \leq 0$, or when the age of the agent $\left(a_{i}\right)$ exceeds $d$ (representing the maximum age threshold), the agent dies. On the other hand, when $E_{i} \geq 2 s$, where $s$ is the basic stored energy, the agent will have the opportunity to reproduce with probability $r$. When reproduction occurs a new agent is created with the same trait as the parent (which assumes unbiased vertical transmission), stored energy equal to $s$ and $a=0$; at the same time the parent's $E$ is reduced by an amount equal to $s$. In the S-mode model, when resource overexploitation occurs traits with lower $f$ are more likely to have $\phi-c$ close to or below zero, which will increase the effect of natural selection by reducing the likelihood of reproduction and increasing mortality. In the case of the I-mode model, trait payoffs exhibit internal negative frequency dependence, so it is possible for a trait with a higher expected payoff $(f)$ to produce lower $\phi$ as a result of trait-specific overexploitation, thereby potentially producing a smaller payoff than an alternative trait with lower $f$ but which is not affected by overexploitation.

In order to maximise $\phi$ each agent $i$ can change its strategy via cultural transmission, which is triggered with a fixed probability $z$ and involves a twostep process. First, an individual $m$ with the highest $\phi$ among $k$ randomly sampled agents is chosen as a model. Then, if $\phi_{i}<\phi_{m}$, the focal agent copies the trait adopted by the model agent. This process is very similar to the model proposed by Shennan [25], although we use the simple expedient of fixing $k$ to 150 , based on Dunbar's number [6]. Clearly, when the population size, $n$, is below $k$ then the latter will be set to $n$.

Individual agents innovate novel traits with frequency $\mu$. When the simulation is initialised, a trait space of length $\theta$ is created. This represents the possible range of traits $j=1 \ldots j=\theta$ that an agent can adopt, each with its own expected payoff $f_{j}$ and, in the case of the I-mode model, a resource pool of size $K_{j}$. When innovation occurs, the focal agent adopts a new trait whose index is the index of the old trait plus the floor of a randomly generated number from a reflected exponential distribution with rate parameter $\lambda$. This model of innovation is close to that previously proposed by Shennan [25], and assumes that innovation:-

1. Is path dependent, so that the current trait determines the range of traits that might be adopted subsequently via innovation;

2. Is stepwise, with frequent small changes and less frequent large changes;

3. Is bi-directional, so that there is a possibility of adopting maladaptive traits (i.e. $\delta$ could be negative); 
4. Can be repetitive, in that the same trait can be 'invented' on multiple occasions by the same or different agents.

\section{Experimental Design}

Every simulation is initialised with 100 agents, each with a randomly assigned age $a$ between 0 and $0.5 d$, a stored energy equal to $s$ and an initial trait set to $41{ }^{1}$ For both S-mode and I-mode models, the trait-space had $\theta=141$, with mean payoff ranging between $8.0\left(f_{1}\right)$ and $15.0\left(f_{141}\right)$, with an interval of 0.05 . In the S-mode model all traits shared a common resource pool of size $K=5000$, while in the I-mode model each independent resource pool $K_{j}$ had an equal size of 1000. All simulations were run for 1000 time-steps, of which the first 200 were dedicated to the simulation 'warm-up'. During this stage innovation and cultural transmission were disabled, allowing the model to reach an equilibrium population size and to allow the creation of different life-histories among the agents, which provided more realistic variability in the system. In the S-mode model we explored the variation of parameters $z, \mu$ and $v$, while for the I-mode we swept only $z$. Exact values of the parameter sweep can be found in table 1. For both modes we conducted 50 simulation runs for each combination of parameters, which we consider sufficient on the basis of an incremental convergence test of up to 500 runs.

Kandler and Laland [12] discuss several possible measures of trait diversity. We use Simpson's diversity Index ( $\tilde{D})$, which takes account of the relative frequency with which those traits occur, such that the state of maximum diversity $(\tilde{D}=1)$ is one in which all possible traits are present in equal proportion, while the minimum diversity $(\tilde{D}=0)$ is one in which only one trait is adopted. This seems to us preferable to a simple trait count because from an evolutionary perspective the diversity of a population in which some traits occur with very low frequency is likely to be less robust than the diversity of a population in which the frequency of traits is more evenly distributed.

\section{Results}

\subsection{S-mode}

Figure 1 records the change in trait diversity over time under regimes of natural selection alone $(z=0.0)$ and two different rates of cultural transmission $(z=0.2$ and $(z=1.0)$. The plot depicts the results from individual simulations because averaging across multiple runs masks the interesting periodicities, but the dynamics are very representative of the full set of results from 50 runs. In the long-run natural selection alone produces high and essentially constant trait diversity $(\tilde{\mathrm{D}}>0.8)$, whereas cultural transmission produces strong fluctuations.

\footnotetext{
${ }^{1}$ Preliminary runs of the model have shown that with the parameter combination specified in table 1 and no innovation, when $j=41$ (and $f_{41}=10$ ) the population grows to an equilibrium around the size set at initialisation.
} 
When all agents rely on cultural transmission at every time-step $(z=1.0)$ diversity is generally very low, albeit punctuated by very brief periods of moderate diversity, derived by episodes of innovation in a relatively low population. When agents are less reliant on cultural transmission $(z=0.2)$ the major fluctuations in diversity are of greater magnitude and persist for longer.

Figure 2 shows the same data depicted in figure 1 for $z=0.2$ between $t=300$ and $t=500$, thereby allowing closer investigation of the dynamics of one cycle of oscillation in diversity. Figure 2a clearly illustrates the fluctuation in Simpson's diversity, while figure $2 \mathrm{~b}$ presents a violin plot of the actual trait distribution. Given that successively numbered traits have higher expected payoff $(f)$, it can be seen from figure $2 \mathrm{~b}$ that 'better' traits were ultimately adopted. It is also clear, however, that this expected improvement was not gradual, but strongly punctuated, such that before most of the agents adopted a novel trait with higher expected payoff there was an intermediate episode of a relatively higher diversity where the trait distribution was multimodal, with intermediate traits between the peaks ignored by the majority of the individuals. Specifically, in this case, at $t=300-340$ the majority of the agents had adopted either trait 41 or 44 , and very few had adopted the intermediate traits 42 and 43 . Subsequently, at circa $t=340$, almost the entire population adopted trait 44 and this remained the predominant choice until $t=450$. Interestingly, during this interval of low trait diversity, several individuals innovated and temporarily adopted traits that were expected to have a higher payoff than trait 44, but these were not adopted by the majority of the population and were soon abandoned by the innovators themselves. From $t=450$, trait 47 became increasingly common, leading again to a bimodal distribution similar to the one seen around $t=300$.

The punctuated dynamics shown in figures 1 and 2 can be explained by the combination of the payoff variance (parameter $v$ ) and the sampling effect due to the size of the sub-population $k$. Suppose that at a given moment in time the entire population has adopted trait $A$. Now suppose an individual agent innovates and adopts the trait $B$, with $f_{B}>f_{A}$. This means trait $B$ produces on average a higher payoff than trait $A$ and should therefore be adopted. At this point an agent who decides to copy the most successful trait will sample $k$ individuals from the population and, if it is lucky, the innovator will be among the $k$ individuals. However, the likelihood that the innovator will be chosen as the model depends not only $k$, but also the payoff variance $v$. If $v$ is high enough, there is a real chance that some agents with trait $A$ will obtain a higher payoff than the innovator's, so the innovation will not be adopted. In the same way, the innovator itself may abandon the new trait and revert back to $A$. In order to allow the population to adopt trait $B$, the difference between $f_{B}$ and $f_{A}$ should be sufficiently high to reduce the effect of $v$, and at the same time, the rate of cultural transmission should not be too high in order to allow the new trait to spread - either by vertical transmission (reproduction) or cultural transmission - before the innovator reverses back to $A$.

From an archaeological perspective the fluctuations in diversity evident in figures 1 and 2 would only be visible in the - perhaps all too rare cases for which high resolution time-series data is available. In many cases archaeological 
sequences suffer from numerous gaps and/or units of observation that are of unequal chronological duration, with the result that assemblages of artefacts or other material often aggregate the results of more than one stage in the evolution of the phenomenon being studied. For this reason it may be helpful to consider the overall frequency with which the evolving system exhibits different degrees of diversity. In figure 3 we follow Kandler and Laland [12] in plotting the frequency distribution of different values of diversity, in this case for natural selection $(z=$ 0 ) and four different rates of cultural transmission $(z>0)$. It is clear that the system is mostly in a state of very high diversity $(\tilde{\mathrm{D}}>0.8)$ when trait evolution is driven by natural selection alone or a lower rate of cultural transmission, and that the reverse is true when the rate of cultural transmission is higher $(\tilde{\mathrm{D}}<0.1)$. The more uniform, but nevertheless weakly bimodal distribution ${ }^{2}$ of diversity recorded for $\mathrm{z}=0.1$ is consistent with the larger and more persistent fluctuations in diversity associated with an intermediate rate of cultural transmission (see figure 1).

Having established the basic dynamic of trait evolution, we now turn to the question of the impact of innovation rate $(\mu)$, rate of cultural transmission $(z)$ and payoff variance $(v)$ on the rate of cultural evolution ${ }^{3}$. The frequency distribution (among 50 simulation runs) of the median trait at the final time-step provides a measure of how far the agents have explored the trait space in the time available (figure 4). When $v=0$ and thus when there is no stochastic component in the payoff evaluation, higher transmission rates produce a considerably higher rate of cultural evolution. When $\mu=0.005$, the highest possible trait (141) is adopted by time-step $t=1000$ in almost all simulations, except for cases where cultural transmission was low or absent $(z<0.1)$. When the rate of innovation is higher $(\mu=0.05)$ this pattern is even more evident, with trait 141 being adopted in almost all simulations even when $0<z>0.1$. This result is consistent with analytical models (e.g. [3]) which predict that the coupling of natural selection and social learning should increase the rate of cultural evolution.

More interesting here, however, is the different result obtained when $v>0$. The first point to note is that in all instances the median terminal trait is considerably lower, with trait 141 never reached. Second, in some cases the relation between $z$ and the terminal median trait appears to be non-monotonic. The positive correlation between the two, which was evident when $v=0$, only holds for low values of $z$, after which increasing the rate of cultural transmission has a negative effect on the evolution of traits offering higher mean payoff. The

\footnotetext{
${ }^{2}$ The presence of a decrease in the frequency at ca $\tilde{\mathrm{D}}=0.02$ for certain values of $z$ (e.g. $z=0.01,0.1$ and 0.3$)$ is caused by the discrete nature of the simulation and the rate of innovation. For instance, suppose that the population size is 500 and all agents have the same trait: when innovation occurs, an average of 5 individuals will adopt a novel trait, which means that in most cases $\tilde{\mathrm{D}}$ will be 0.0198 , and smaller values can be obtained only when the number of innovator is less than 5 .

${ }^{3}$ We use 'rate of cultural evolution' to refer to the rapidity with which the trait space is explored. In adopting this terminology we are, however, aware that although higher $j$ potentially yield higher payoffs, this does not guarantee that agents with higher $j$ are reproductively fitter (e.g. if there is overexploitation), and nor do we mean to imply that cultural evolution is necessarily a fitness enhancing process.
} 
point of inflection where the effect of $z$ is reversed depends on the combination of $\mu$ and $v$ and is quite marked in some cases (e.g. $\mu=0.05$ and $v=1$ ) and barely perceptible in others $(\mu=0.005$ and $v=3)$. These results are consistent with the dynamics we suggested above. High enough rates of cultural transmission can cause an innovator to revert to an earlier trait before the true mean payoff of the novel trait has been established, whereas a lower rate of cultural transmission reduces the likelihood that the innovator will engage in social learning, thereby prolonging the opportunity for the innovator to both reproduce and act as a model for other agents. Both the latter processes can increase the frequency of the novel trait, which will in turn reduce the sampling effect described above. The results also clearly demonstrate that an increase in the innovation rate has the opposite effect of an increase in payoff variance, so the optimal rate of cultural transmission is ultimately determined by the interaction between both factors. This result provides a formal demonstration of the argument [19, 21] that the optimal balance of individual learning and social learning should shift towards the former as the environment becomes less predictable.

\subsection{I-mode}

When the adoption of novel traits facilitates the exploitation of different independent resources, the advantage deriving from adopting a trait with a higher mean payoff $f$ is potentially diminished or negated once the sum of payoffs sought by agents who have adopted that trait exceeds the size of the resource pool associated with it (i.e. $\Omega_{j}>K_{j}$ ). Recall that this situation is modelled by equation 1 applied to each trait in turn, thus if there are two traits $A$ and $B$, with $f_{A}<f_{B}$ and $K_{A}=K_{B}$, then so long as $\Omega_{B}<K_{B}$ the payoff obtained by agents adopting trait $B$ will on average be larger than that obtained by those adopting $A$. When this condition is not satisfied, however, then the average $\phi$ of agents adopting trait $A$ could be higher if $\Omega_{A}<K_{A}$, potentially leading to a reversion to trait $A$ as a result of cultural transmission. When multiple traits are overexploited, the term $\frac{\omega_{i}}{\Omega}$ in equation 1 approximates to the reciprocal of the number of agents who have adopted the particular trait $\left(1 / n_{j}\right)$, thus the difference in $\phi$ becomes primarily a function of $K_{j}$ and $n_{j}$ rather than $f_{j}$. It follows that, under conditions of overexploitation, all $n$ agents will receive the same payoff provided that the number adopting each trait is proportional to the size of the resource pool associated with that trait. One would thus expect the long-term equilibrium distribution of traits to be one in which all traits are adopted in proportion to the size of their associated resource pool. This is also the expectation of the Ideal Free Distribution (IFD) model [29, 32], which predicts that the stable equilibrium for resource exploitation in a given environment is one in which the number of individuals occupying each subsistence niche is proportional to the quantity of resources available in that niche.

There is, however, a fundamental difference between our model and the Ideal Free Distribution model: the 'ideal' assumption is violated by the fact that our agents do not have perfect information about the value of different traits, and hence they have to rely on innovation and cultural transmission to 
explore the trait-space. This difference derives from our interest in the evolution of subsistence strategies: the fact that subsistence trait diversity is likely to be a function of the preceding history of subsistence technology as well as the kinds of rational decision-making captured by the diet-breadth model and the IFD model. In other words, given the factors influencing the innovation and spread of traits, it is not self-evident that the expectations of models such as the IFD will always be met, and even if they are, there may be unexpected dynamics en-route to the predicted long-term equilibrium.

In order to investigate the temporal pattern of trait adoption (niche exploration), we investigate trait richness in terms of the width of the envelope containing all traits from that offering the lowest payoff to that offering the highest payoff. Figure 5a depicts the time-series of the upper and lower-bound of the trait distribution for two different rates of cultural transmission, $z$, and reveals several interesting results. First, in both cases, traits offering lower payoff are lost through time. Second, the width of the trait envelope associated with the two different values of $z$ is very different. Third, the lower rate of cultural transmission produces faster cultural evolution in the sense that it leads to the more rapid adoption of traits offering a higher payoff. Figure 5b extends the analysis to additional values of $z$ by plotting the width of the associated trait envelope at three moments in time $(t=400,700$ and 1000). This reveals a non-monotonic relation between $z$ and the rate of cultural evolution. Increasing the rate of cultural transmission from $z=0$ to $z=0.05$ increases the speed with which the population 'discovers' the trait offering the highest payoff, although it also reduces the number of traits that are maintained in the population. Further increase in $z$ reduces the rate of cultural evolution, to the extent that for $z>0.2$ the trait offering the highest payoff is not discovered within 1000 time-steps; it also narrows the trait envelope to the point where it remains constant in width $(z=1.0)$.

The process responsible for the pattern depicted in figure 5 can be explored by plotting the changing population size of the most prevalent traits during a single run of the simulation. Figure 6 illustrates this for $z=0.5$ in a narrow temporal window of 40 time-steps. The population curves reveal continuous switching back and forth between two traits, initially 48 and 49 and then, after a brief appearance by trait 51 , traits 49 and 52 . What we see here is the effect of excessive reliance on cultural transmission: thus trait 49 is initially the most common, but the payoff declines as this 'niche' fills up, eventually leading more agents to revert back to trait 48 , which has lower $f$ but will, for the reasons discussed above, have higher $\phi$. Then, as more agents switch to trait $48, \Omega_{48}$ becomes larger and $\Omega_{49}$ smaller, resulting in a relative increase in $\phi_{49}$ and a renewed preference for trait 49 . This cyclical pattern is eventually interrupted by the emergence of trait 51, after which there is a period of fluctuation between trait 49 and 52. It appears that the effect of cultural transmission on this dynamic is as follows. When $z$ is relatively low the opportunity to switch traits is reduced, with the result that traits offering lower payoff are always 'maintained' by a subset of the population. These traits will in fact be continuously re-adopted whenever a novel trait with higher $f$ is being overexploited. 
Consequently, lower $z$ promotes both the continuous adoption of traits with lower $f$ and the adoption of novel higher $f$ traits without the risk of reversion and subsequent loss from the population. Conversely, higher $z$ increases the opportunity for reversion, thereby narrowing the width of the trait envelope and increasing the possibility of trait loss, thus ultimately reducing the rate of cultural evolution.

The pattern of alternation between two traits, involving reversion to traits with lower $f$ as a consequence of resource overexploitation, has been predicted by several optimal foraging theoretic models $[1,31]$, although these assume both full knowledge of the available resources and the possibility of exploiting multiple resources. Our model adds an understanding of how adaptive trait switching in a population is mediated by the rate of cultural transmission. In particular, our results suggest that the rate of cultural transmission has a significant effect on the likelihood of obtaining the Ideal Free Distribution in situations where the exploitation of new resources requires innovation.

\section{Conclusions}

Our S-mode conceptual model shares many similarities with Kandler and Laland's [12] recent model of trait evolution, so it is reassuring that implementations using different modelling paradigms (a reaction-diffusion model and an agent-based simulation) should yield some similar results, even though one incorporates 6 traits (Kandler and Laland) and the other 141 traits (our model). Like Kandler and Laland, we find that adaptive traits that exploit the same, shared, resource undergo punctuated evolution: periods of low diversity and little or no change in trait distribution are interrupted by periods of higher diversity during which the distribution typically becomes multimodal before coalescing around a new trait with higher expected payoff. We also find that the rate of cultural transmission strongly influences the amplitude and frequency of changes in diversity.

Nevertheless, the motivation for our work is different from Kandler and Laland's in that we are primarily concerned with the evolution of particular class of adaptive trait: subsistence strategies. In our introduction we noted the power of diet-breadth models to explain diversity in subsistence strategies, but also asked how cultural evolutionary processes might promote or retard the ability of populations to achieve predicted outcomes. For this reason we created a model which allowed us to study the efficacy of cultural evolution for improving payoff, how this is influenced by payoff variance and how the evolutionary dynamics differ for traits that facilitate the exploitation of different independent resources rather than one shared resource. We draw the following conclusions from our model:-

1. The amount of payoff variance substantially alters the frequencies of cultural transmission and innovation required to effect the rapid cultural evolution of adaptive traits. Higher rates of cultural transmission are advantageous when there is zero payoff variance, but in the presence of even 
modest payoff variance they are deleterious in the sense that, after a given duration, the population may have adopted traits that yield lower payoff than would have been the case had trait adoption been driven by natural selection alone. However, increasing the innovation rate enhances the efficacy of cultural transmission when payoff variance is high.

2. When payoff variance is non-zero the relationship between the rate of cultural transmission and the rate of cultural evolution of adaptive traits (i.e. the speed with which the trait offering the highest payoff is 'discovered') is not monotonic. The lowest and highest rates of cultural transmission retard the rate of cultural evolution relative to intermediate rates of cultural transmission.

3. Rapid cultural evolution is associated with variability in trait diversity. Permanently low trait diversity, caused by the highest rates of cultural transmission, and permanently high diversity, caused by the lowest rates of cultural transmission, are both associated with slower adoption of traits offering higher expected payoff.

4. The distribution of adaptive traits that exploit independent resources evolves to a state close to the theoretical Ideal Free Distribution only if the rate of cultural transmission is relatively low. Higher rates of cultural transmission reduce trait diversity, so that a more limited number of 'niches' are occupied at any given time. We have demonstrated that this effect is a direct result of excessive reliance on cultural transmission, which results in increased episodes of trait reversion and ultimately trait loss.

Although we are confident in the conclusions drawn from our model, we are aware that there are plausible alternative assumptions under which they might not hold. First, we explain conclusions 1 and 2 in terms of sampling error causing the early loss of innovations. This is a function of payoff variance, but also the size of the subpopulation, $k$, that provides potential models for cultural transmission. A different value of $k$, or the imposition of demic or network structure, or a different mode of cultural transmission might all alter the outcome.

Second, the strict resource independence of traits in the I-mode model should be considered a limiting case, since in many real world contexts some degree of inter-relationship between traits is likely, such that overexploitation in one niche is likely to at least partially affect the availability of resources in others.

Third, our model assumes that each agent adopts a single trait. This is a widely accepted convention in models of cultural transmission, but is at odds with behavioural ecology models such as the diet breadth model, where multiple resources are exploited by single individuals. Whether allowing individuals to adopt multiple traits ultimately alters population-level trait diversity is a question which merits further investigation. 
Finally, one could argue that the S-mode and I-mode models are not really mutually exclusive, but represent different stages in the hierarchical exploration of trait space. In this case I-mode innovation opens up new subsistence niches while S-mode innovation improves exploitation within an existing niche, rather as Boden [2] differentiated between 'impossibilist' creativity and 'improbabilist' creativity. The two types of innovation will be structured in a hierarchically and nested trait-space, where path-dependence will determine different evolutionary trajectories [4]. A given S-mode innovation designed to exploit a specific type of resource might in fact enhance or inhibit - depending on its intrinsic properties - the probability of an I-mode innovation.

Notwithstanding the scope for further investigation, our model demonstrates how payoff variance, the rate of cultural transmission and the innovation rate combine to alter the rate of cultural evolution of adaptive traits, and thus to promote or retard the ability of populations to achieve optimal diversity in subsistence strategies.

\section{Acknowledgements}

We gratefully acknowledge the use of the UCL Legion High Performance Computing Facility, and associated support services, in the completion of this work. The AHRC Centre for Evolution of Cultural Diversity (grant APN111956) kindly covered our computing costs. Enrico Crema is supported by a UCL Graduate School Scholarship. We are particularly grateful to Stephen Shennan and James Steele for their encouragement, and to Anne Kandler for her detailed comments on the direction of this research. We would also like to thank the three anonymous reviewers for their careful scrutiny of our manuscript and their helpful suggestions. 


\section{Tables}

Table 1: Model parameters, default values and sweep range. $z$ swept in S-mode for $0,0.01,0.025,0.05,0.075,0.1,0.125,0.15,0.175,0.2,0.25,0.3,0.5$ and 1.0. $z$ swept in I-mode for $0.0,0.01,0.05,0.1,0.2,0.3,0.5$ and 1.0. $f$ swept with interval of 0.05 .

\begin{tabular}{ccccc} 
Parameter Name & Abbreviation & Default Value & Sweep (S-Mode) & Sweep (I-Mode) \\
\hline Initial Number of Agents & $N$ & 100 & - & - \\
Reproduction Rate & $r$ & 0.2 & - & - \\
Resource Consumption & $c$ & 8 & - & - \\
Basic Storage & $s$ & 20 & - & - \\
Maximum Age & $d$ & 20 & - & - \\
Innovation Rate & $\mu$ & 0.05 & $0.005,0.05$ & - \\
Rate Parameter of Innovation Step & $\lambda$ & 1 & - & - \\
Maximum number of observed agents & $k$ & 150 & - & - \\
Cultural Transmission Rate & $z$ & - & $0.1-1.0$ & - \\
Shared Resource Pool Size & $K$ & 5000 & - & - \\
Independent Resource Pool Size & $K$ & 1000 & - & - \\
Trait Average Payoff & $f$ & $8-15$ & - & - \\
Trait Variance & $v$ & 1 & $0,1,3$ & - \\
Length of Trait Space & $\theta$ & 141 & - & - \\
Initial Trait & - & 41 & - & - \\
Time-steps & $T$ & 1000 & - & \\
Duration of Warmup Phase & $W$ & 200 & - & \\
\hline
\end{tabular}


Figures 


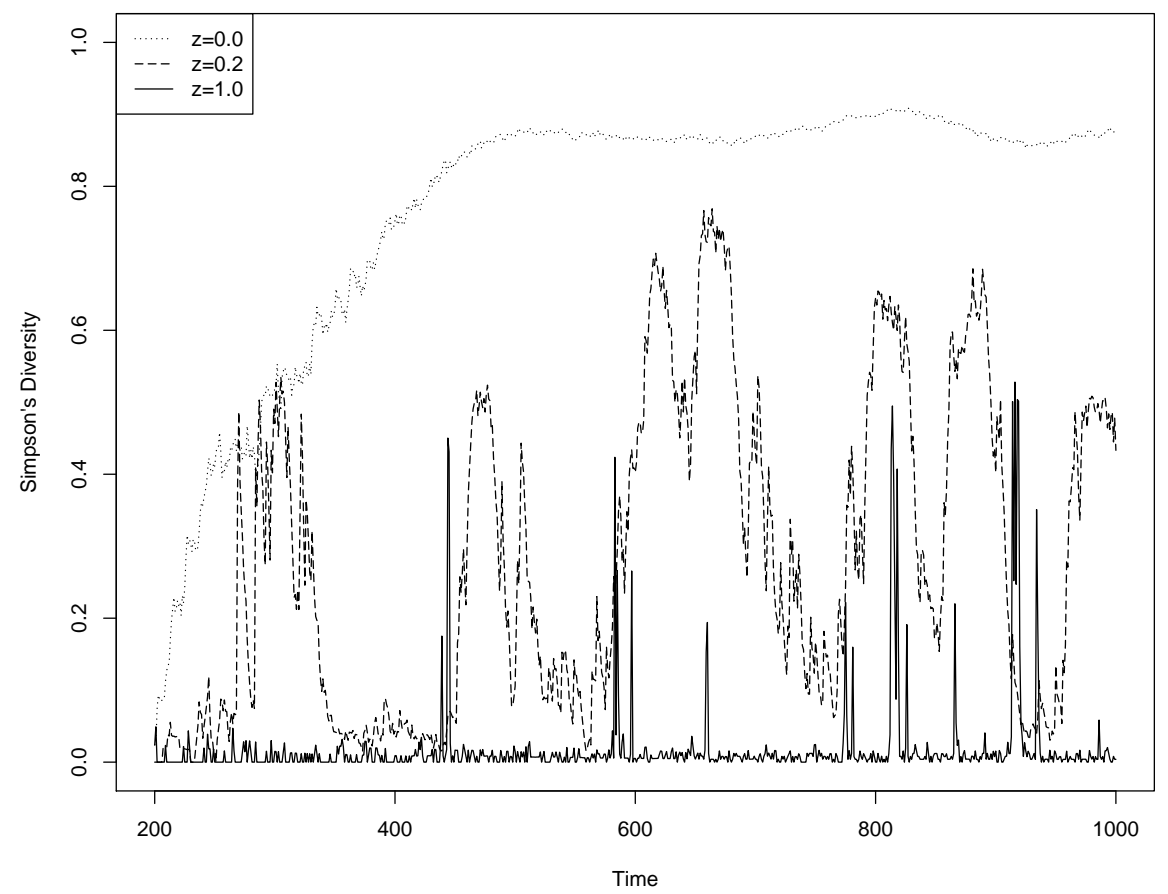

Figure 1: Change in trait diversity over time for single simulations each with a different rate of cultural transmission, $z(v=1, r=0.2, \mu=0.01$. $)$ 

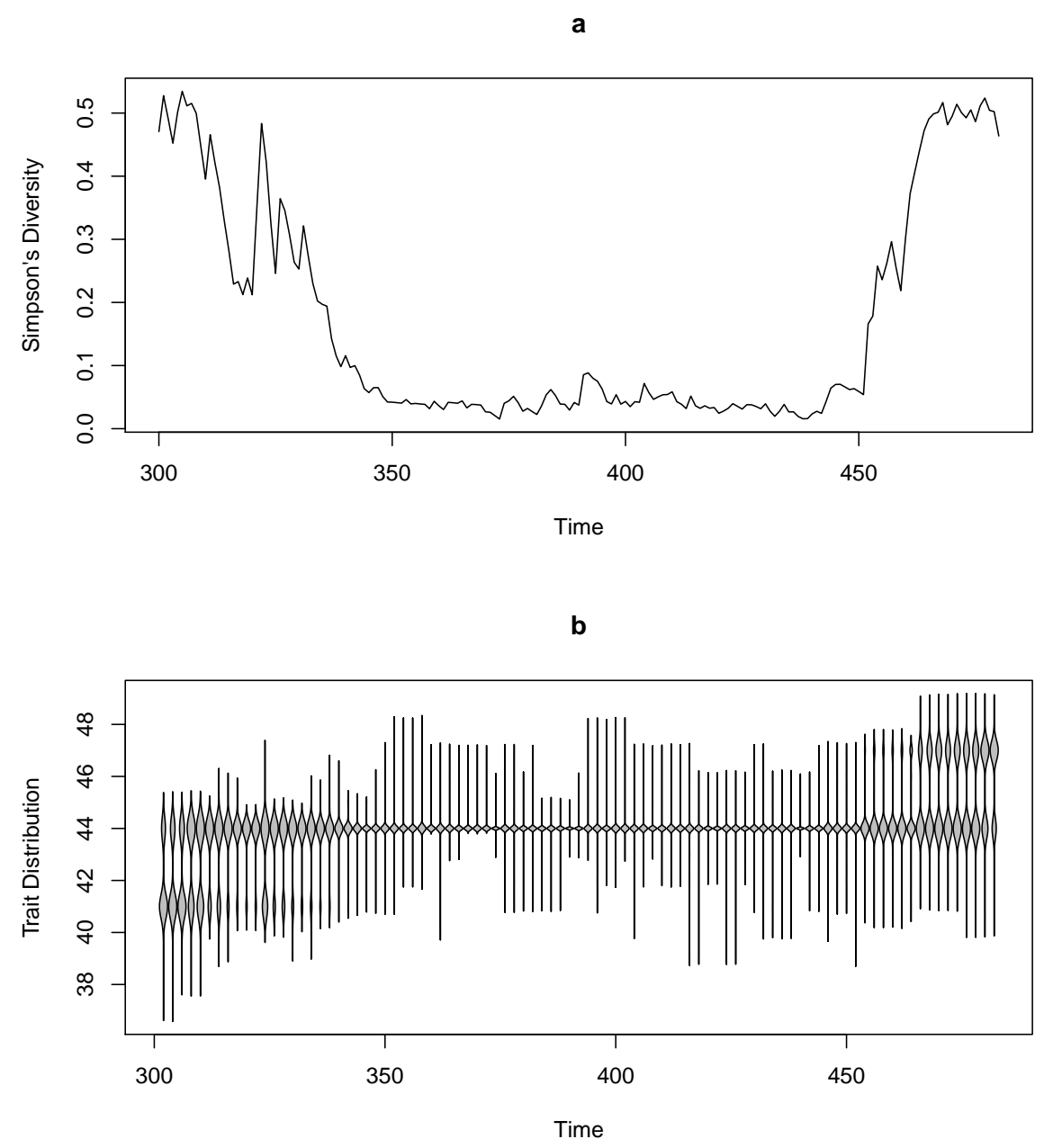

Figure 2: Change in trait diversity from $t=300$ to $t=500$ for a single simulation with $z=0.2$, displayed as: a) a plot of Simpson's Index; b) a violin plot of the trait distribution. 


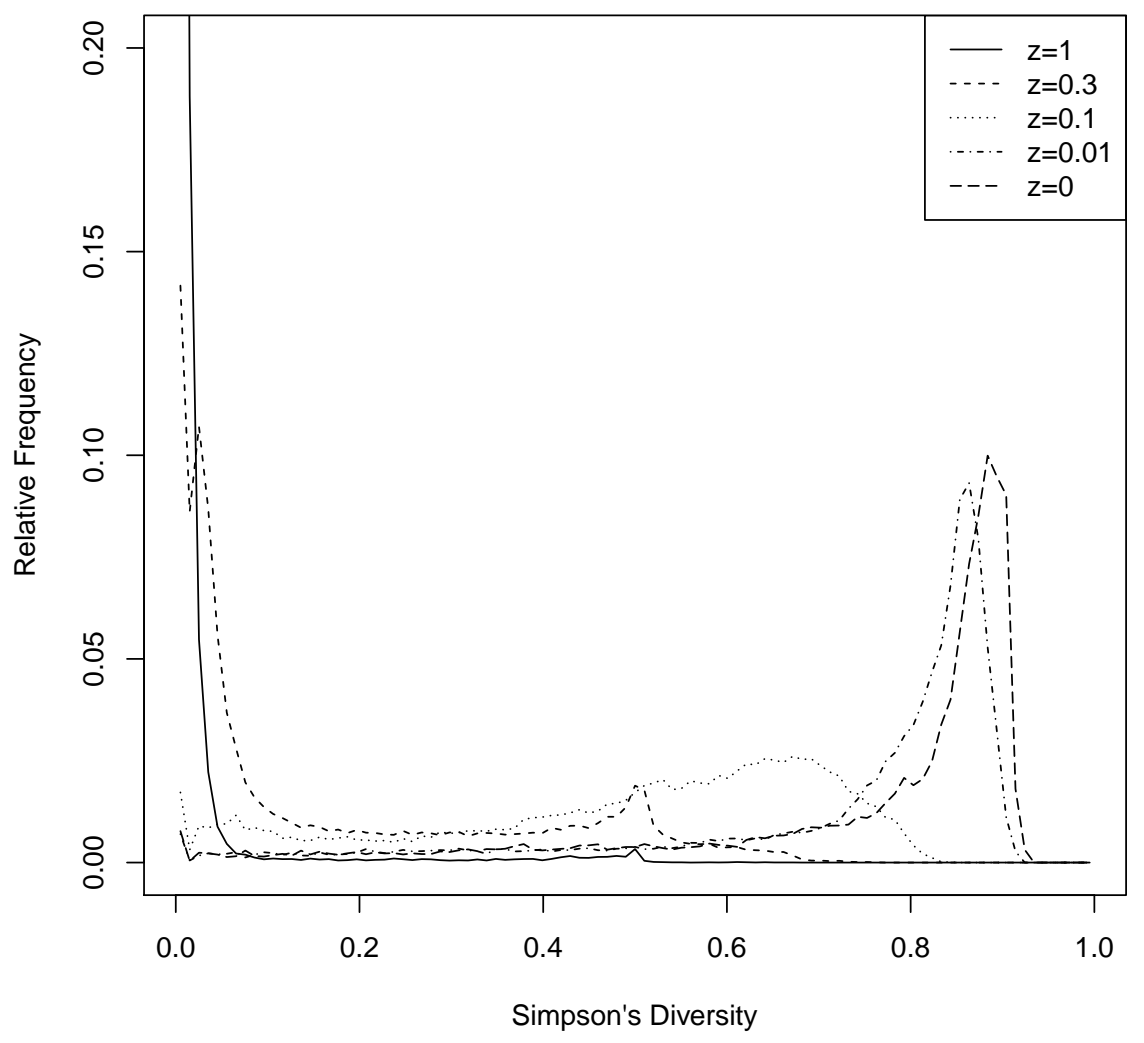

Figure 3: Frequency distribution of the amount of diversity, as measured by Simpson's Index, for 5 different rates of cultural transmission, $z(\mu=0.01$, $v=1$ and $r=0.2$; frequency derived from a total of 50 runs, with bin interval of 0.01$)$. 

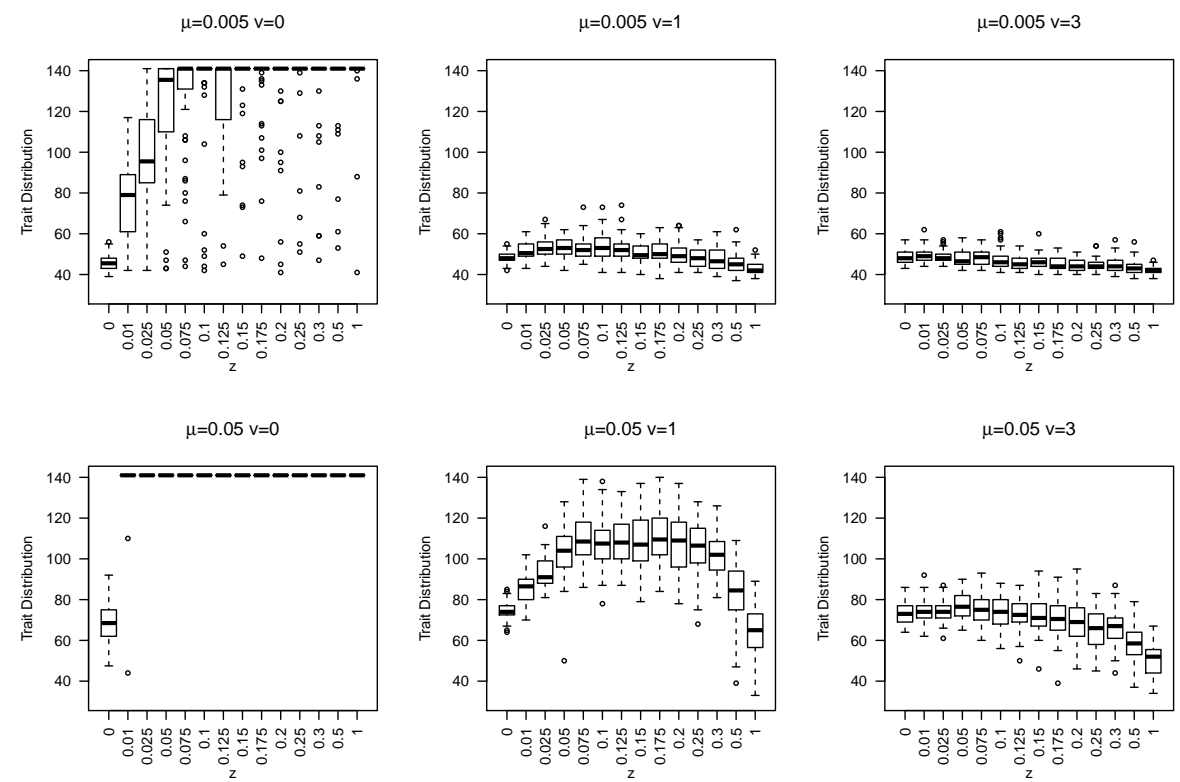

Figure 4: Distribution of the terminal median trait for 14 different rates of cultural transmission, $z$, with 2 rates of innovation, $\mu$, and 2 amounts of payoff variance, $v$. ( $r=0.2$; total of 50 runs. $)$ 

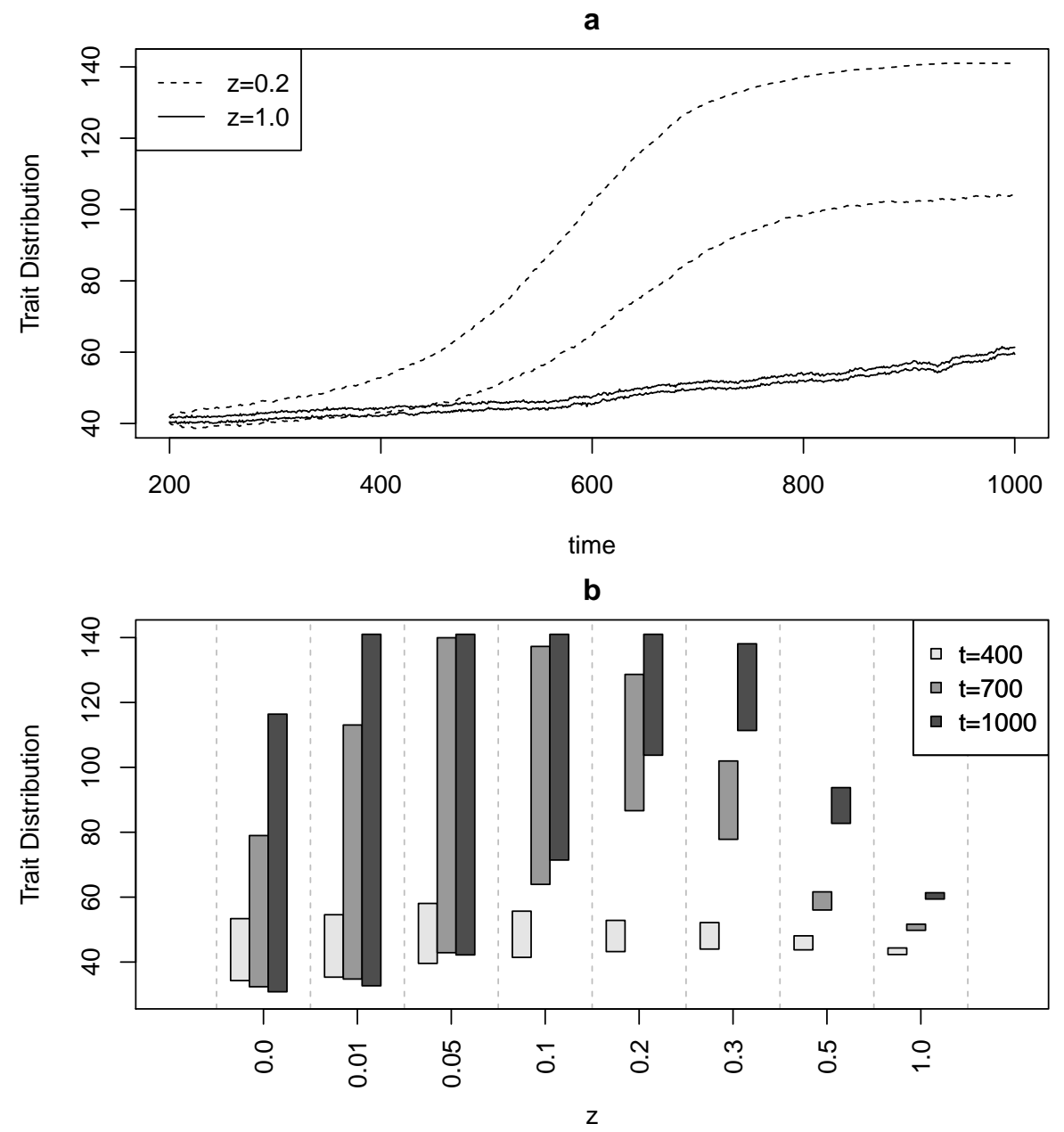

Figure 5: (a) The upper and lower bound of the trait envelope for 2 different rates of cultural transmission, $z$. (b) The width of the trait envelope at $t=400$, 700 and 1000 for 8 different rates of cultural transmission, $z$. $(v=1, r=0.2$, $\mu=0.05$ and $K=1000$ for each trait. The results are from 50 runs of the simulation.) 


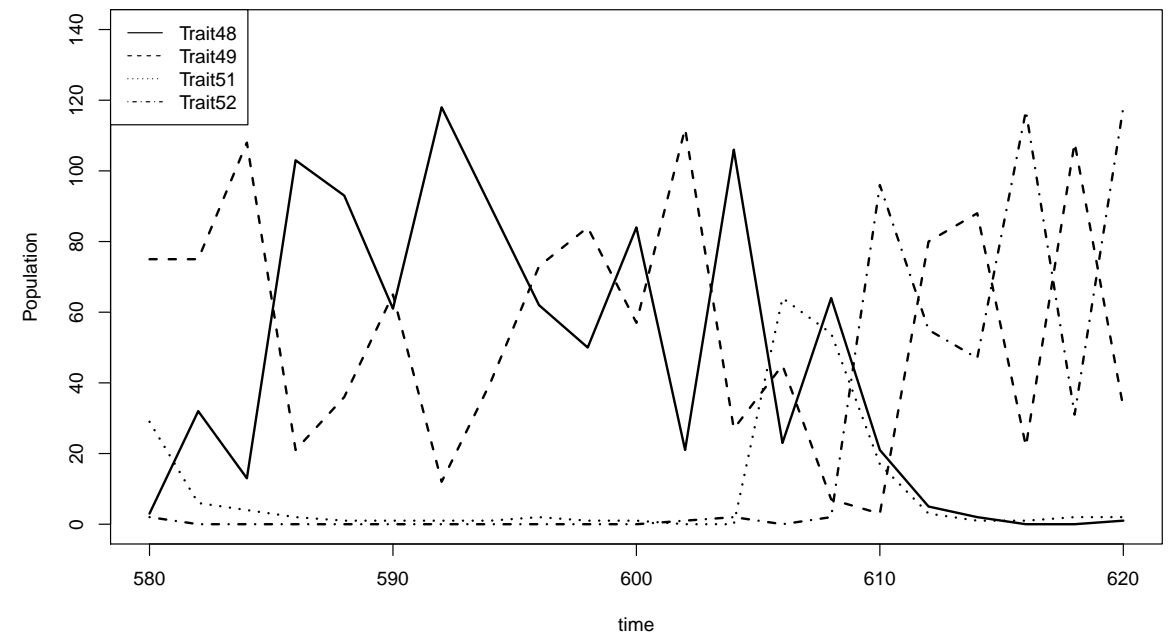

Figure 6: The population size of the four most prevalent traits during a 40 timesteps interval from a single run. With $z=0.5, \mu=0.05, r=0.2$, and $K=1000$ for each trait. 


\section{Supplementary material: ODD Protocol of the ABM}

The following model description is based on the ODD (Overview, Design, Detail) protocol described by Grimm and colleagues $[8,9]$.

\section{PURPOSE}

The purpose of the model is to explore the spread and the distribution of adaptive traits via model-biased cultural transmission. Although the basic structure of the model remains constant, two different modes have been created based on whether different traits exploit independent resource pools ( $I$-mode) or a single shared resource pool ( $S$-mode). Both models were specifically designed to explore the effects of innovation, population dynamics, frequency dependence and stochasticity in the distribution and diversity of traits adopted by a population.

\section{ENTITIES, STATE VARIABLES, AND SCALES}

The model consists of $n$ agents, each possessing four state variables: age $(a)$, amount of stored energy $(E)$, most recent payoff $(\phi)$ and adopted trait $(j)$. The first three variables are updated at each time-step, while the adopted trait can change only through innovation or cultural transmission. The model is tuned by the following set of parameters:

- Fitness related parameters:

- Rate of reproduction (range:0-1,r)

- Amount of resource consumption $(c)$

- Initial/Basic stored amount of energy $(s)$

- Age limit $(d)$

- Innovation related parameters:

- Innovation rate (range:0-1, $\mu$ )

- Rate parameter of innovation step $(\lambda)$

- Cultural transmission related parameters

- Rate of cultural transmission (range:0-1,z)

- Maximum number of observed agents $(k)$

- Trait-space related parameters

- Trait index $(j)$

- Length of trait-space (number of traits, $\theta$ )

- Trait average payoff $\left(f_{j}\right)$

- Trait variance $(v)$

- Shared resource pool size (for the S-mode models, $K$ )

- Trait-specific resource pool size (for the I-mode models, $K_{i}$ ) 
- Trait adopted at initialisation $(\alpha)$

- General parameters

- Number of agents at initialisation $(N)$

- Length of the warm up stage $(w)$

- Overall duration of each run (i.e. number of time-steps, $T$ )

\section{PROCESS OVERVIEW AND SCHEDULING}

The model proceeds through a sequence of time-steps ( 1 to $T$ ) where decision making and state variables are updated. The first $W$ time-steps are dedicated to the 'warm-up' of the model, where the agent will neither innovate nor copy other agents trait/strategy and will instead only proceed through the following sequence of sub-processes: (1) Ageing; (2) Death; (3) Reproduction; and (4) Payoff Evaluation. After $W$ time-steps, this sequence will be updated with two new sub-processes as follow: (1) Ageing; (2)Death; (3)Reproduction; (4) Innovation; (5) Cultural Transmission; and (6) Payoff Evaluation.

\section{DESIGN CONCEPTS}

\section{Basic Principles}

The model implements a variant of the model-biassed transmission and innovation proposed by Shennan [25]. Most differences have marginal impacts (e.g. using a reflected exponential distribution instead of a reflected gamma distribution), however the critical difference is determined by the fact that the adoption of a trait determines the reproductive fitness of its owner (thus agents can die and/or reproduce), the payoff derived by the trait is stochastic and it has a negative frequency dependence triggered in case of overexploitation.

\section{Emergence}

The dynamics of change in the distribution of traits along with changes in population sizes are all emergent properties of the system derived by the decision making of each agent.

\section{Adaptation}

The agent adapts to the environment by modifying their trait through cultural transmission and innovation.

\section{Objectives}

The main objective of each agent is to maximise the payoff obtained each time-step. This will lead to an improvement of its reproductive fitness.

\section{Learning}

The agents can modify their traits either by innovation (with frequency $\mu$ ) or by social learning (with frequency $z$ ). The former will 
determine the unconditional adoption of a novel trait, which could be equally deleterious or advantageous. The latter will involve a direct comparison of the focal agents' foraging success and a model agent's success, with the latter being the individual with the highest current payoff among $k$ randomly sampled individuals from the population. The trait of the model agent will be copied only if its payoff is higher than the one of the focal agents. Furthermore agents do not possess memory of traits adopted previously, nor do they recall the past evaluations of observed samples. Thus agents might adopt the same trait multiple times through re-innovation or cultural transmission.

\section{Sensing}

The agents have complete knowledge of recent payoff and strategy of $k$ random agents every time a successful draw from a Bernoulli distribution with probability $z$ occurs. Notice also that the sampled agents will be different each time.

\section{Interaction}

No direct interaction between individuals occurs. However agents can indirectly determine the reduction in the payoff other agents when overexploitation occurs.

\section{Stochasticity}

Stochastic components are widely present in the model. These are:

(a) definition of the agents' state variable $a$ at the initialisation as a random draw from an uniform distribution (bounded by 0 and $0.5 d)$

(b) bernoulli draws for triggering innovation and cultural transmission (with success probabilities $\mu$ and $z$ ),

(c) random sampling of the observed agents (with size $k$ ),

(d) payoff evaluation (random draw from a normal distribution with mean $f_{j}$ and standard deviation $v$ ),

(e) exploration of the trait space through innovation (random draw from a discrete reflected exponential distribution, with rate parameter $\lambda$ ).

\section{Collectives}

Strictly speaking no collectives are present in the model. However when recent payoff is evaluated in the I-mode model, agents possessing the same trait are treated as a group exploiting the same niche. This means that in case of overexploitation, the reduction in the payoff is restricted to individuals exploiting the same trait.

\section{Observations}

During each simulation the following data is collected:

(a) total number of agents,

(b) number of individuals adopting each existing trait,

(c) highest and lowest trait adopted by the population, 
(d) median trait,

(e) simpson's diversity index (D̃) of the traits given by:

$$
\tilde{\mathrm{D}}=1-\sum_{j=1}^{\theta} p_{j}^{2}
$$

where $\theta$ is the total number of possible traits, and $p_{j}$ is the relative proportion of trait $j$.

\section{INITIALISATION}

The model is initialised by the creation of $N$ agents, with each agent $i$ having a random age $a$ between 0 and $0.5 d, E_{i}=s$, and the initial trait $\alpha$.

\section{INPUT DATA}

No external time-varying data are input in the model.

\section{SUB-MODELS}

\section{Reproduction and Death}

Reproduction occurs at frequency $r$ when the agent's storage $(E)$ is at least twice the initial storage value $s$. When a child is created, the parent's storage is reduced by $s$. The child will have the parent's trait (which mimics vertical transmission), have $a=0$ and $E=s$. Death will occur when the agent's age is greater than $d$, or when $E \leq 0$.

\section{Payoff Evaluation}

The payoff $\left(\phi_{i}\right)$ for an agent $i$ is evaluated as follow. First, the contribution $\omega_{i}$ of each agent is defined as a random draw from a normal distribution with mean $f_{j}$ and standard deviation $v$ where $j$ is an index referring to a position in the trait space. We then define:

$$
\Omega=\sum_{j=1}^{\theta} \Omega_{j}
$$

where $\Omega_{j}$ is the sum of all $\omega_{i}$ of individual adopting the trait $j$.

In the S-mode, $\phi_{i}$ is then computed as follows:

$$
\begin{aligned}
& \text { if } \Omega \leq K, \phi_{i}=\omega_{i} \\
& \text { if } \Omega>K, \phi_{i}=K \frac{\omega_{i}}{\Omega}
\end{aligned}
$$

which essentially leads to a proportional share of the resource pool in case of overexploitation. In the case of I-mode, equation (3) becomes 
trait specific, and thus $\Omega$ is substituted by $\Omega_{j}$, and $K$ by $K_{j}$. Thus in this case the overexploitation of the resource pool affects only the individuals possessing the same trait. Payoff is then reduced by an amount $c$, corresponding to the consumption rate, before being added to stored energy $E$.

\section{Innovation}

Innovation occurs when a bernoulli draw with probability $\mu$ is successful. The agent will change its current trait by 'moving' in the trait space by an amount equal to $\delta$, the floor of a number randomly drawn from a reflected exponential distribution with rate parameter equal to $\lambda$. This means that the majority of innovation will determine small positive or negative move in the trait space, with some rare instance of 'jumps' in both directions occurring with a frequency negatively correlated to $\lambda$.

\section{Cultural Transmission}

Cultural transmission occurs with frequency $z$ and is structured in three parts. First, $k$ random individuals are sampled from the population, and the agent with the highest recent payoff is chosen as a model. Second, the payoff received by the model is compared to the agent's own most recent payoff. Third, depending on the outcome of the comparison, the focal agent will decide to copy (if its $\phi$ is lower than the model's) or to maintain its own trait (if its $\phi$ is higher or equal to the model's). The process can be regarded as guided form of model biased transmission. The actual update of the adopted trait occurs synchronically at the end of the subprocess. This ensures that the agents evaluates the right combination of trait and payoff. In computational terms this is accomplished by generating a copy of all agents to which the actual observation refers to, with the actual updating of the traits occurring to the original set. 


\section{References}

[1] Belovsky, G. E., An optimal foraging-based model of hunter-gatherer population dynamics, Journal of Anthroplogical Archaeology 7 (1988) 329-372.

[2] Boden, M. A., Prècis of "The creative mind: Myths and mechanisms", Behavioral and Brain Sciences 17 (1994) 519-570.

[3] Boyd, R. and Richerson, P. J., Culture and the Evolutionary Process (University Press, Chicago, 1985).

[4] Boyd, R. and Richerson, P. J., How microevolutionary processes give rise to history, in Evolution and History, eds. Niteki, M. and Niteki, D. (State University of New York Press, Albany, 1992), pp. 149-178.

[5] Costopoulos, A., Evaluating the impact of increasing memory on agent behaviour: Adaptive patterns in an agent-based simulation of subsistence, Journal of Artificial Societies and Social Simulation 4 (2001).

[6] Dunbar, R. I. M., Neocortex size as a constraint on group size in primates, Journal of Human Evolution 22 (1992) 469-493.

[7] Epstein, J. M. and Axtell, R., Growing Artificial Societies: Social Science from the Bottom Up (Brookings Press and MIT Press, 1996).

[8] Grimm, V., Berger, U., Bastiansen, F., Eliassen, S., Ginot, V., Giske, J., Goss-Custard, J., Grand, T., Heinz, S. K., Huse, G., Huth, A., Jepsen, J. U., Jørgensen, C., Mooij, W. M., Müller, B., Pe'er, G., Piou, C., Railsback, S. F., Robbins, A. M., Robbins, M. M., Rossmanith, E., Rüger, N., Strand, E., Souissi, S., Stillman, R. A., Vabø, R., Visser, U., and DeAngelis, D. L., A standard protocol for describing individual-based and agent-based models, Ecological Modelling 198 (2006) 115-126.

[9] Grimm, V., Berger, U., DeAngelis, D. L., Polhill, J. G., Giske, J., and Railsback, S. F., The ODD protocol: A review and first update, Ecological Modelling 221 (2010) 2760-2768.

[10] Hames, R. B. and Vickers, W. T., Optimal diet breadth theory as a model to explain variability in Amazonian hunting, American Ethnologist 9 (1982) pp. 358-378.

[11] Hodder, I., Symbols in Action (Cambridge University Press, Cambridge, I982).

[12] Kandler, A. and Laland, K. N., An investigation of the relationship between innovation and cultural diversity, Theoretical Population Biology 76 (2009) 59-67.

[13] Layton, R., Foley, R., and Williams, E., The transition between hunting and gathering and the specialized husbandry of resources: A socio-ecological approach, Current Anthropology 32 (1991) 255-274. 
[14] Lesne, A., The discrete versus continuous controversy in physics, Mathematical Structures in Computer Science 17 (2007) 185-223.

[15] MacArthur, R. and Pianka, E., On the optimal use of a patchy environment, American Naturalist 100 (1966) 603-609.

[16] Mace, R., Transitions between cultivation and pastoralism in Sub-Saharan Africa, Current Anthropology 34 (1993) 363-382.

[17] Mellars, P., The emergence of biologically modern populations in Europe: a social and cognitive 'revolution'?, in Primates and Man, eds. Runciman, W. G., Smith, J. M., and Dunbar, R. I. M. (Clarendon Press, Oxford, 1996), pp. 179-202.

[18] Mithen, S. J., Thoughtful Foragers: A Study of Prehistoric Decision Making (Cambridge University Press, Cambridge, 1990).

[19] Odling-Smee, F. J., Multiple levels in evolution: An approach to the naturenurture issue via applied epistemology, in Animal Models of Human Behaviour, ed. Davey, G. C. L. (John Wiley \& Sons Ltd., 1983), pp. 135-158.

[20] Oota, H., Pakendorf, B., Weiss, G., von Haeseler, A., Pookajorn, S., Settheetham-Ishida, W., Tiwawech, D., Ishida, T., and Stoneking, M., Recent origin and cultural reversion of a hunter-gatherer group., PLoS Biology 3 (2005) e71.

[21] Plotkin, H. C., The Nature of Knowledge: Concerning Adaptations, Instinct and the Evolution of Intelligence (Allen Lane (Penguin), London, 1994).

[22] Pyke, H., Pulliam, H., and Charnov, E., Optimal foraging theory: A selective review of theory and tests, Quarterly Review of Biology 52 (1977) $137-154$.

[23] Schrire, C., An inquiry into the evolutionary status and apparent identity of San hunter-gatherers, Human Ecology 8 (1980) 9-32.

[24] Shennan, S., Evolution in archaeology, Annual Review of Anthropology 37 (2008) 75-91.

[25] Shennan, S. J., Demography and cultural innovation: a model and its implications for the emergence of modern human culture, Cambridge Archaeological Journal 11 (2001) 5-16.

[26] Smith, E. A., Anthropological applications of optimal foraging theory: A critical review, Current Anthropology 24 (1983) 625-51.

[27] Steele, J., Human dispersals: Mathematical models and the archaeological record, Human Biology 81 (2009) 121-140. 
[28] Stephens, D. W. and Krebs, J. R., Foraging Theory (University Press, Princeton, 1986).

[29] Tregenza, T., Building on the ideal free distribution, Advances in Ecological Research 26 (1995) 253-307.

[30] Winterhalder, B., Foraging strategies in the boreal environment: An analysis of Cree hunting and gathering, in Hunter-Gatherer Foraging Strategies: Ethnographic and Archaeological Analyses (University of Chicago Press, Chicago, 1981), pp. 66-98.

[31] Winterhalder, B., Baillargeon, W., Cappelletto, F., I Randolph Daniel, J., and Prescott, C., The population ecology of hunter-gatherers and their prey, Journal of Anthropological Archaeology 7 (1988) 289-328.

[32] Winterhalder, B., Kennett, D. J., Grote, M. N., and Bartruff, J., Ideal free settlement of California's Northern Channel Islands, Journal of Anthropological Archaeology 29 (2010) 469-490. 